\title{
Numerical Solution of Fractional Diffusion Equation Model for Freezing in Finite Media
}

\author{
R. S. Damor, Sushil Kumar, and A. K. Shukla \\ Department of Applied Mathematics and Humanities, S. V. National Institute of Technology, Surat-395 007, India \\ Correspondence should be addressed to R. S. Damor; rameshsvnit2010@gmail.com
}

Received 28 March 2013; Accepted 8 August 2013

Academic Editor: C. Nataraj

Copyright (c) 2013 R. S. Damor et al. This is an open access article distributed under the Creative Commons Attribution License, which permits unrestricted use, distribution, and reproduction in any medium, provided the original work is properly cited.

\begin{abstract}
Phase change problems play very important role in engineering sciences including casting of nuclear waste materials, vivo freezing of biological tissues, solar collectors and so forth. In present paper, we propose fractional diffusion equation model for alloy solidification. A transient heat transfer analysis is carried out to study the anomalous diffusion. Finite difference method is used to solve the fractional differential equation model. The temperature profiles, the motion of interface, and interface velocity have been evaluated for space fractional diffusion equation.
\end{abstract}

\section{Introduction}

Heat diffusion during the change of state, for example, melting and solidification, has important applications in science and technology including casting of nuclear waste materials, vivo freezing of biological tissues, and solar collectors. Problems involving heat conduction in materials in which a phase change occurs are known as moving boundary problems. First time Stefan [1] discussed this problem to study the melting of polar ice, so this is referred as Stefan problem. Solidification is the phase change problem in which phase transformation takes place by descending the energy of the system, that is, heat extraction from the liquid region. The problem related to the heat conduction involving solidification has drawn the attention of many researchers. Solidification process can be grouped into two major categories, that is, solidification of pure substances and alloy. Solidification of pure substances is usually characterized by a sharp solid or liquid interface. On the other hand, a mixed phase region characterizes solidification of an alloy. The mixed phase regions are commonly termed as the mushy region; this is a combination of liquid solute and solid crystals [2].

Fractals and fractional calculus have been used to improve the modelling accuracy of many phenomena in natural sciences. The most important advantage of using fractional differential equations is their nonlocal property.
This means that the next state of a system depends not only upon its current state but also depends upon all of its historical states. These are more realistic and one of reasons to make the fractional calculus more and more popular $[3,4]$. The fractional model is appropriate for modelling complex dynamics as the ions are undergoing anomalous diffusion. Fractional order models are more general and useful than the previously used integer order model. Fractional derivative enables the description of the memory and hereditary inherent properties in various processes governed by anomalous diffusion. Fractional diffusion equations are studied by many researchers. Meerschaert et al. [5] gave a second-order accurate numerical approximation for the fractional diffusion equation, Murio [6] discussed implicit finite difference approximation for time fractional diffusion equation, Lijuan et al. [7] gave finite difference-approximation for the fractional advection diffusion equation using RiemannLiouville space fractional derivative. Fractional differential equation models are used to study anomalous diffusion; when a space fractional derivative is replaced by the second-order derivative in a diffusion model, it leads to super-diffusion [8].

Some researchers have studied simple or classic Stefan problem using fractional diffusion equation. Li et al. [9] gave some exact solution to Stefan problem by adopting Caputo form of fractional time derivative and Dirichlet boundary conditions. Voller [10] obtained an exact solution 
of a Stefan problem by considering Stefan number as zero. Recently, Atkinson [11] wrote a very interesting survey paper on moving boundary problems for time and space fractional diffusion. They studied the classic Stefan problem and discussed analytical or semianalytical solution in closed form for infinite or semi-infinite region.

Alloy solidification problems are distinct from classic or simple Stefan problem as the melting temperature is not known in advance; this depends on the composition of the alloy [12]. Since in alloy phase change occurs on wide range, the mathematical formulations are highly nonlinear as given by Carslaw and Jaeger [13]. A numerical solution can be obtained using finite difference method. The enthalpy method is based on weak formulation, which was used by many researchers (Alexiades and Solomon [14], Shamsunder and Sparrow [15], Voller [16], Bonacina et al. [17], Hoffman and Bischof [18], Jiji and Gaye [19], and Kumar and Katiyar $[2,20])$. The essential features of the basic enthalpy methods are that they do not need tracking of the solid-liquid interface; a fixed grid can be used for numerical computations, and the nonlinearity at the moving boundary can also be avoided.

In present study, the enthalpy formulation of space fractional diffusion model is proposed to study the alloy solidification in finite media. Space fractional derivative of order $\alpha \in(1,2]$ is considered of the Riemann-Liouville form, and it is approximated by shifted Grunwald formula [3]. Finite difference method is used to obtain numerical solution of the model. To study the effect of fractional order derivative $\alpha$, the temperature profiles and motion of freezing interface are obtained for different values of $\alpha$.

1.1. Used Fractional Derivative. Fractional derivative is denoted as ${ }_{a} D_{t}^{\alpha} f(t)$; the subscripts $a$ and $t$ denote the two limits related to the operation of fractional differentiation, which is called the terminal of fractional differentiation. The negative values for $\alpha$ are denoted for fractional integrals of arbitrary order.

\subsection{Riemann-Liouville Fractional Derivative [3]. Consider}

$$
\begin{aligned}
& { }_{a} D_{t}^{\alpha} f(t) \\
& = \begin{cases}\frac{d^{m}}{d t^{m}}\left[\frac{1}{\Gamma(m-\alpha)} \int_{a}^{t} \frac{f(\tau)}{(t-\tau)^{\alpha+1-m}}\right], & \text { for } m-1<\alpha<m, \\
\frac{d^{m}(f(t))}{d t^{m}}, & \text { for } \alpha=m .\end{cases}
\end{aligned}
$$

\subsection{Shifted Grunwald Approximation [3]. Consider}

$$
\begin{aligned}
\frac{\partial^{\alpha} T(x, t)}{\partial x^{\alpha}}= & \frac{1}{\Gamma(-\alpha)} \lim _{M \rightarrow \infty} \frac{1}{h^{\alpha}} \\
& \times \sum_{k=0}^{M} \frac{\Gamma(k-\alpha)}{\Gamma(k+1)} T(x-(k-1) h, t),
\end{aligned}
$$

$$
\begin{aligned}
\frac{\partial^{\alpha} T(x, t)}{\partial x^{\alpha}} \approx & \frac{1}{h^{\alpha}} \sum_{k=0}^{i+1}\left(g_{k}\right) T_{i-k+1}^{n}, \\
& \text { where } g_{k}=\frac{1}{\Gamma(-\alpha)} \frac{\Gamma(k-\alpha)}{\Gamma(k+1)}
\end{aligned}
$$

\section{Model Description}

One-dimensional geometry is considered as shown in Figure 1. A molten material with initial temperature $T_{0}$ and uniformly distributed volumetric heat generation $q$ is confined between two surfaces $x=0$ and $x=l$. At $x=0$, convecting cooling is applied to the mould at temperature $T_{\infty}$, and the surface $x=l$ is taken as adiabatic.

\section{Mathematical Model}

3.1. Governing Equation. We propose the governing fractional diffusion equation model for alloy solidification as follows.

In solid region

$$
\rho_{s} c_{s} \frac{\partial T_{s}}{\partial t}=k_{s} \frac{\partial^{\alpha} T_{s}}{\partial x^{\alpha}}+q, \quad 1<\alpha \leq 2,0 \leq x \leq S(t) .
$$

In liquid region

$$
\rho_{l} \mathcal{c}_{l} \frac{\partial T_{l}}{\partial t}=k_{l} \frac{\partial^{\alpha} T_{l}}{\partial x^{\alpha}}+q, \quad 1<\alpha \leq 2, S(t) \leq x \leq l .
$$

The conditions at the moving interface $x=S(t)$ are given as

$$
\begin{gathered}
k_{s} \frac{\partial^{\alpha-1} T_{s}}{\partial x^{\alpha-1}}-k_{l} \frac{\partial^{\alpha-1} T_{l}}{\partial x^{\alpha-1}}=\rho L \frac{d S(t)}{d t} \quad \text { at } x=S(t), \\
T_{s}=T_{l}=T_{\mathrm{ph}} \quad \text { at } x=S(t),
\end{gathered}
$$

where $\rho, c, k, T$, $t$, and $x$ represent density, specific heat, thermal conductivity, temperature, time, and distance, respectively. Moreover, $S(t), q$, and $L$ are denoted for position of phase change interface, volumetric heat generation, and latent heat, respectively. The subscript $s, l$, and ph are concerned for the solid state, liquid state, and phase change, respectively.

On making the use of enthalpy

$$
H(T)=\int_{T_{0}}^{T} c d T
$$

where $T_{0}$ is the reference temperature, (3) to (5) become

$$
\rho \frac{\partial H}{\partial t}=K(x, t) \frac{\partial^{\alpha} T(x, t)}{\partial x^{\alpha}}+q, \quad 0 \leq x \leq l .
$$




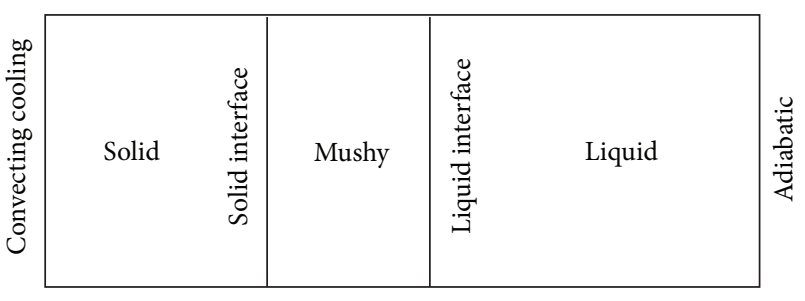

FIgURE 1: Schematic of the physical problem.

The relation between enthalpy and temperature is given as $[17,18]$

$H(T)$

$$
= \begin{cases}c_{s}\left(T-T_{m s}\right), & \text { for } T<T_{m s}, \\ \left(T-T_{m s}\right)\left(\frac{c_{s}+c_{l}}{2}+\frac{L}{T_{m l}-T_{m s}}\right) & \text { for } T_{m s} \leq T \leq T_{m l}, \\ L+\frac{1}{2}\left(c_{s}+c_{l}\right)+c_{l}\left(T-T_{m l}\right), & \text { for } T>T_{m l},\end{cases}
$$

and thermal conductivity is given as

$$
K= \begin{cases}k_{s} & \text { for } T<T_{m s} \\ \frac{k_{s}+k_{l}}{2} & \text { for } T_{m s} \leq T \leq T_{m l} \\ k_{l}, & \text { for } T>T_{m l}\end{cases}
$$

where $T_{m l}$ and $T_{m s}$ are liquidus and solidus temperature, respectively.

3.2. Initial and Boundary Conditions. (i) Initially, the system is at $255^{\circ} \mathrm{C}$; that is,

$$
T(x, t)=255^{\circ} \mathrm{C}, \quad \text { at } t=0 .
$$

(ii) Convective boundary condition is at $x=0$; that is,

$$
K \frac{\partial T(x, t)}{\partial x}=h\left(T(x, t)-T_{\infty}\right), \quad \text { at } x=0,
$$

where $h$ is the heat transfer coefficient and $T_{\infty}$ is the temperature of the cooling media.

(iii) Adiabatic condition is at $x=l$; that is,

$$
\frac{\partial T(x, t)}{\partial x}=0, \quad \text { at } x=l \text {. }
$$

\section{Numerical Solution}

Using forward difference for time derivative and shifted Grunwald approximation [3] for space fractional derivative, finite difference scheme for (8) is given as

$$
\begin{aligned}
H_{i}^{n+1} & =H_{i}^{n}+\frac{\Delta t}{(\Delta x)^{\alpha} \rho_{i}^{n}} K_{i}^{n} \sum_{k=0}^{i+1}\left(g_{k}\right) T_{i-k+1}^{n}+\frac{\Delta t}{\rho_{i}^{n}} q_{i}^{n}, \\
{[H]^{n+1} } & =[H]^{n}+\frac{\Delta t}{(\Delta x)^{\alpha}}[G]^{n}[T]^{n}+\Delta t\left[\frac{q}{\rho}\right]^{n},
\end{aligned}
$$

TABLE 1: Thermophysical properties of $\mathrm{Sn}-5 \mathrm{wt} \% \mathrm{~Pb}[21]$.

\begin{tabular}{lc}
\hline Properties & Value \\
\hline Thermal conductivity of solid $\left(\mathrm{W} / \mathrm{m}^{\circ} \mathrm{C}\right)$ & 65.6 \\
Thermal conductivity of liquid $\left(\mathrm{W} / \mathrm{m}^{\circ} \mathrm{C}\right)$ & 32.8 \\
Density of solid $\left(\mathrm{kg} / \mathrm{m}^{3}\right)$ & 7475 \\
Density of liquid $\left(\mathrm{kg} / \mathrm{m}^{3}\right)$ & 7181 \\
Specific heat of solid $\left(\mathrm{J} / \mathrm{kg}^{\circ} \mathrm{C}\right)$ & 217 \\
Specific heat of liquid $\left(\mathrm{J} / \mathrm{kg}^{\circ} \mathrm{C}\right)$ & 253 \\
Latent heat $(\mathrm{KJ} / \mathrm{kg})$ & 592.14 \\
Solidus temperature $\left({ }^{\circ} \mathrm{C}\right)$ & 183 \\
Liquidus temperature $\left({ }^{\circ} \mathrm{C}\right)$ & 224.9 \\
\hline
\end{tabular}

where

$$
\begin{aligned}
& G(i, j)=0 \quad \text { if } j \geq i+2, \\
& G(i, j)=\frac{K_{i}}{\rho_{i}} g_{0} \quad \text { if } j=i+1, \\
& G(i, j)=\frac{K_{i}}{\rho_{i}} g_{1} \quad \text { if } j=i, \\
& G(i, j)=\frac{K_{i}}{\rho_{i}} g_{i-j+1} \quad \text { if } j \leq i-1 .
\end{aligned}
$$

Here $i$ and $n$ are space and time steps, respectively. Space and time increments are denoted by $\Delta x$ and $\Delta t$, respectively. These are adjustable subjects to satisfy the stability criteria, as $\Delta t /(\Delta x)^{\alpha} \leq 1 / d_{\max } \alpha$, where $d_{\max }$ is the maximum value for thermal diffusivity. For $\alpha=2$, this stability criterion is reduced to $\Delta t /(\Delta x)^{2} \leq 1 / d_{\max } 2$ [18]. On calculating the enthalpy at $(n+1)$ th level using (14), temperature distribution at $(n+1)$ th time level is calculated by reverting $(9)$. Once the new temperature field is obtained from enthalpy, the process repeats until the system reaches steady state. Isotherms at $224.9^{\circ} \mathrm{C}$ and $183.0^{\circ} \mathrm{C}$ give the position of liquidus and solidus interface, respectively.

\section{Results and Discussion}

Thermophysical properties of the $\mathrm{Sn}-5 \mathrm{wt} \% \mathrm{~Pb}$ alloy used for simulations are enlisted in Table 1 [21]. To study the transient behaviour of the system, the whole process of solidification is divided into four different stages as in $[2,22]$.

In stage 1, the entire region is in liquid state; this stage ends when the mushy region starts at $x=0$. During stage 2 , there is the coexistence of liquid and mushy regions; this stage ends when the entire region converts into mushy region. Stage 3 begins when solidification takes place from $x=0$; during this stage, the entire region may be in the solid-mushy or solidmushy-liquid state. This stage ends when the entire region is solidified. Only solid phase exists in stage 4, which ends when the system reaches the steady state. In our study, we fix the values of $q=500 \mathrm{~W} / \mathrm{m}^{3}$ and $h=1500 \mathrm{~W} / \mathrm{m}^{2}$ and observe the temperature profiles, liquidus interface, solidus interface, and rates of liquidus, and solidus interface for different values of $\alpha$. 


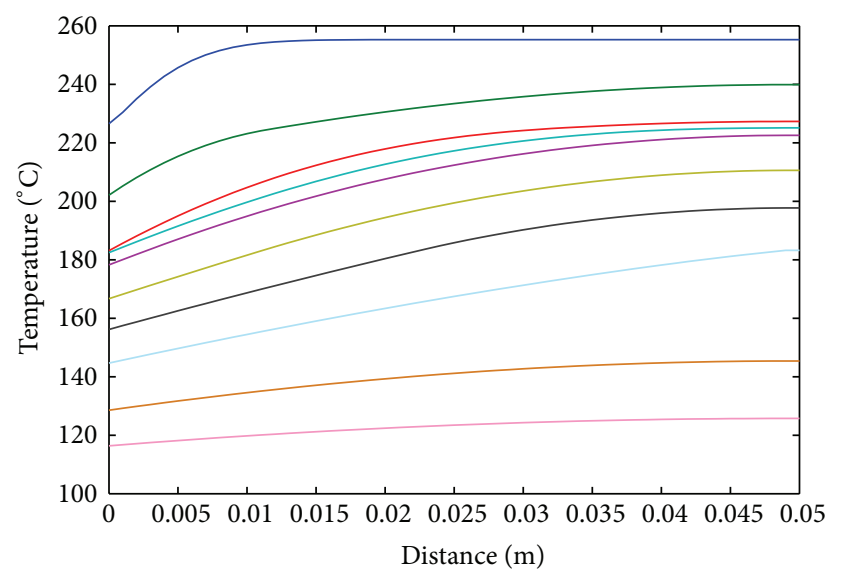

$\begin{aligned}-t & =1 \mathrm{~s} & -t & =2000 \mathrm{~s} \\ -t & =100 \mathrm{~s} & -t & =3000 \mathrm{~s} \\ -t & =460 \mathrm{~s} & -t & =3779 \mathrm{~s} \\ -t & =679 \mathrm{~s} & -t & =3850 \mathrm{~s} \\ t & =1000 \mathrm{~s} & -t & =4265 \mathrm{~s}\end{aligned}$

Figure 2: Temperature profile in region at different time $t$ for $\alpha=2$.

Figure 2 shows the temperature profile for $\alpha=2$ at $t=1$ second, $t=100$ seconds, $t=460$ seconds, $t=679$ seconds, $t=1000$ seconds, $t=2000$ seconds, $t=3000$ seconds, $t=$ 3779 seconds, $t=3850$ seconds, and $t=4265$ seconds. Stage 1 ends at $t=1$ second when the mushy state starts at $x=0$. At $t=100$ second the system is in stage 2 ; that is, the mushy and liquid phases coexist. Stage 2 ends at $t=679$ seconds, while stage 3 starts at $t=460$ seconds. Here stage 3 starts before the end of stage 2. At $t=1000$ seconds, there exist solid mushy and liquid states. At $t=3000$ seconds, the solid and mushy phases coexist; that is, the system is in stage 3. Stage 3 ends at $t=3779$ seconds, when the entire region is solidified. At $t=3850$ seconds, the system is now in stage 4 , which ends at $t=4265$ seconds when the steady state is obtained.

Figure 3 shows the temperature profile for $\alpha=1.9$ at $t=$ 0.3 seconds, $t=36$ seconds, $t=100$ seconds, $t=277$ seconds, $t=800$ seconds, $t=1100$ seconds, $t=1378$ seconds, $t=$ 1420 seconds, $t=1500$ seconds, and $t=1812$ seconds. Here, stage 1 ends at $t=0.3$ seconds, when the mushy state starts at $x=0$. At $t=100$ seconds, the system turns in stage 2 ; that is, the region where mushy and liquid phases coexist. Stage 2 ends at $t=277$ seconds, and the process of stage 3 starts at $t=36$ seconds. Here stage 3 starts before the end of stage 2 . At $t=800$ seconds, system is in solid-mushy-liquid states. At $t=1100$ seconds, the solid and mushy phases coexist; that is, the system enters in stage 3 . Stage 3 ends at $t=1378$ seconds, when the entire region converts into the solid state. At $t=$ 1420 seconds and $t=1500$ seconds, the system is now in stage 4 , which ends when the steady state is obtained at $t=1812$ seconds.

Figure 4 shows the temperature profile for $\alpha=1.8$ at $t=$ 0.3 seconds, $t=34$ seconds, $t=100$ seconds, $t=274$ seconds, $t=1000$ seconds, $t=1500$ seconds, $t=2084$ seconds, $t=$ 2120 seconds, $t=2200$ seconds, and $t=2703$ seconds. Stage 1 ends at $t=0.3$ seconds, when the mushy state starts at $x=0$.

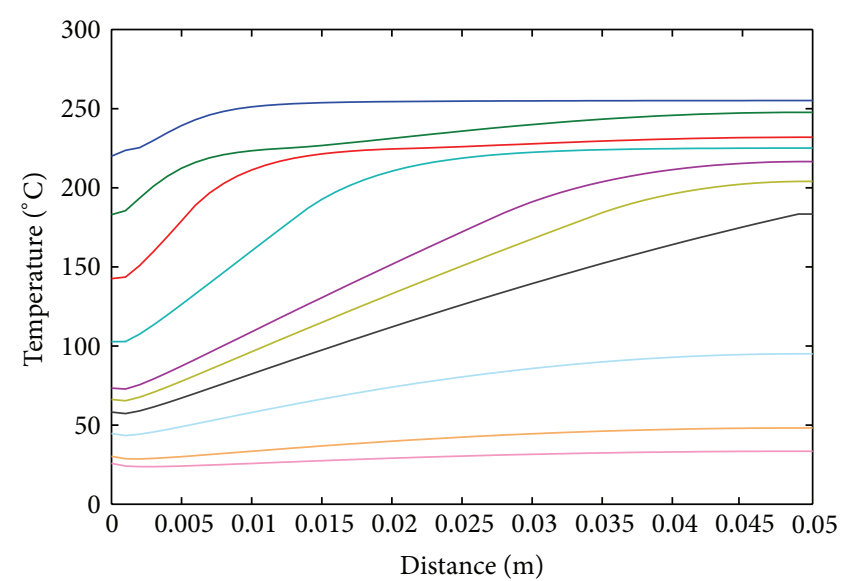

$\begin{aligned}-t & =0.3 \mathrm{~s} \\ t & =36 \mathrm{~s} \\ t & =100 \mathrm{~s} \\ t & =277 \mathrm{~s} \\ t & =800 \mathrm{~s}\end{aligned} \quad \begin{aligned}-t & =1100 \mathrm{~s} \\ -t & =1420 \mathrm{~s} \\ -t & =1500 \mathrm{~s} \\ -t & =1812 \mathrm{~s}\end{aligned}$

FIgURE 3: Temperature profile in region at different time $t$ for $\alpha=$ 1.9 .

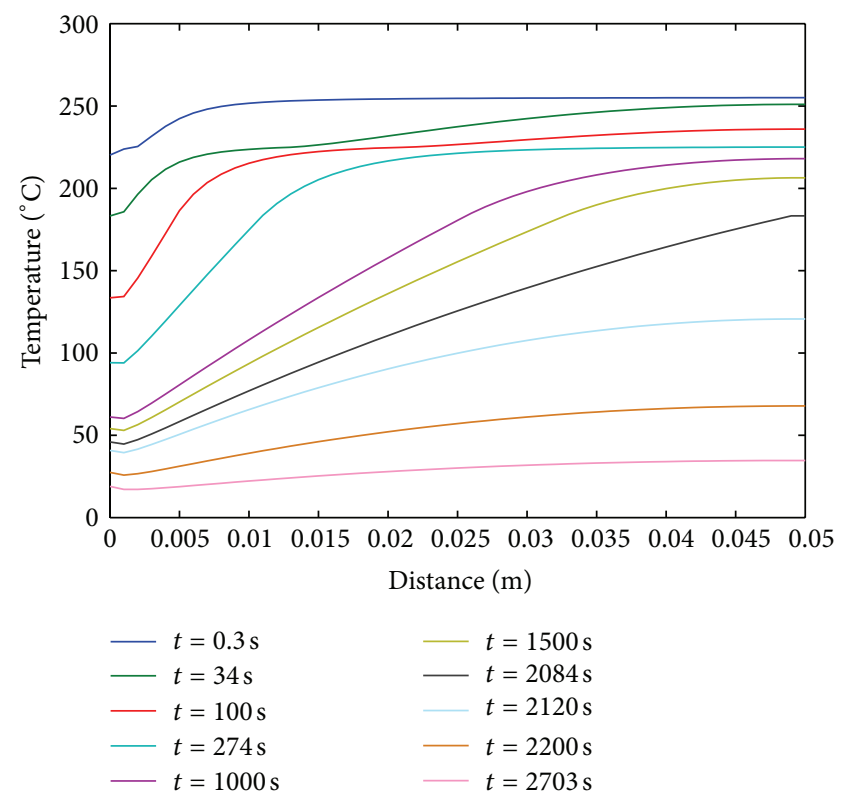

Figure 4: Temperature profile in region at different time $t$ for $\alpha=$ 1.8 .

At $t=100$ seconds, the system enters into stage 2 ; that is, the coexistence of mushy and liquid phases. Stage 2 ends at $t=$ 274 seconds, while stage 3 starts at $t=34$ seconds. Here the stage 3 starts before the end of stage 2 . At $t=1000$ seconds, there exist solid mushy and liquid states. At $t=1500$ seconds, we observe the solid and mushy phases; that is, the system is now at stage 3. Stage 3 ends at $t=2084$ seconds, when solidification takes place in the entire region. At $t=2120$ seconds and $t=2200$ seconds, the system enters in stage 4 , which ends when it attains its steady state at $t=2703$ seconds. 
TABle 2: Time taken to the end of the stages for different $\alpha$.

\begin{tabular}{lcccc}
\hline Alpha & Stage 1 & Stage 2 & Stage 3 & Stage 4 \\
\hline$\alpha=2$ & $t=1$ second & $t=679$ seconds & $t=3779$ seconds & $t=3850$ seconds \\
$\alpha=1.9$ & $t=0.3$ seconds & $t=277$ seconds & $t=1378$ seconds & $t=1812$ seconds \\
$\alpha=1.8$ & $t=0.3$ seconds & $t=274$ seconds & $t=2084$ seconds & $t=2703$ seconds \\
$\alpha=1.7$ & $t=0.5$ seconds & $t=537$ seconds & $t=3805$ seconds & $t=4739$ seconds \\
$\alpha=1.6$ & $t=1$ second & $t=1804$ seconds & $t=8837$ seconds & $t=10247$ seconds \\
\hline
\end{tabular}

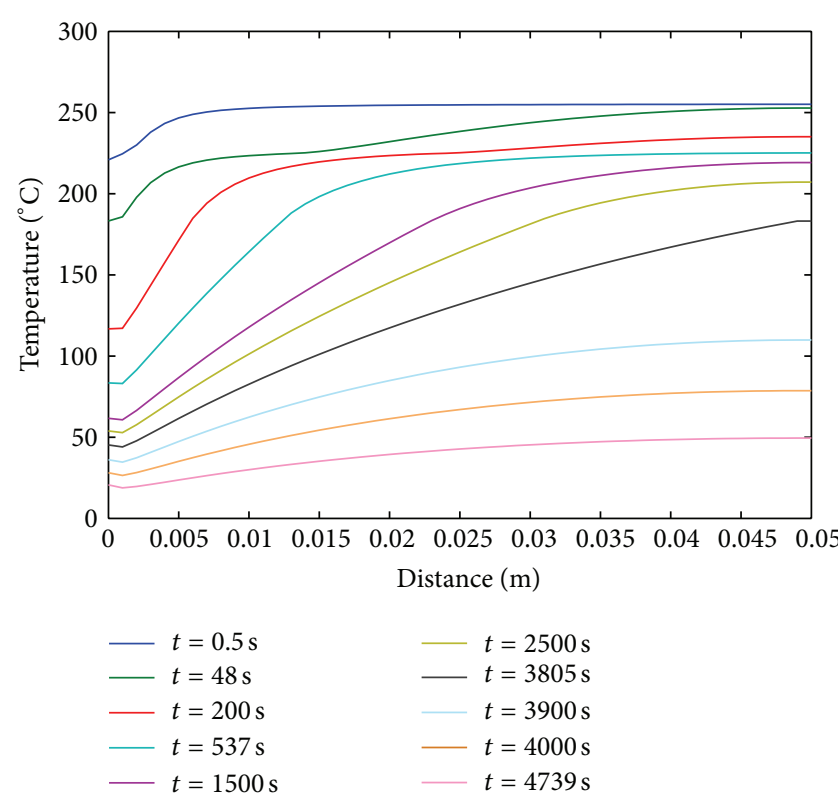

FIGURE 5: Temperature profile in region at different time $t$ for $\alpha=$ 1.7.

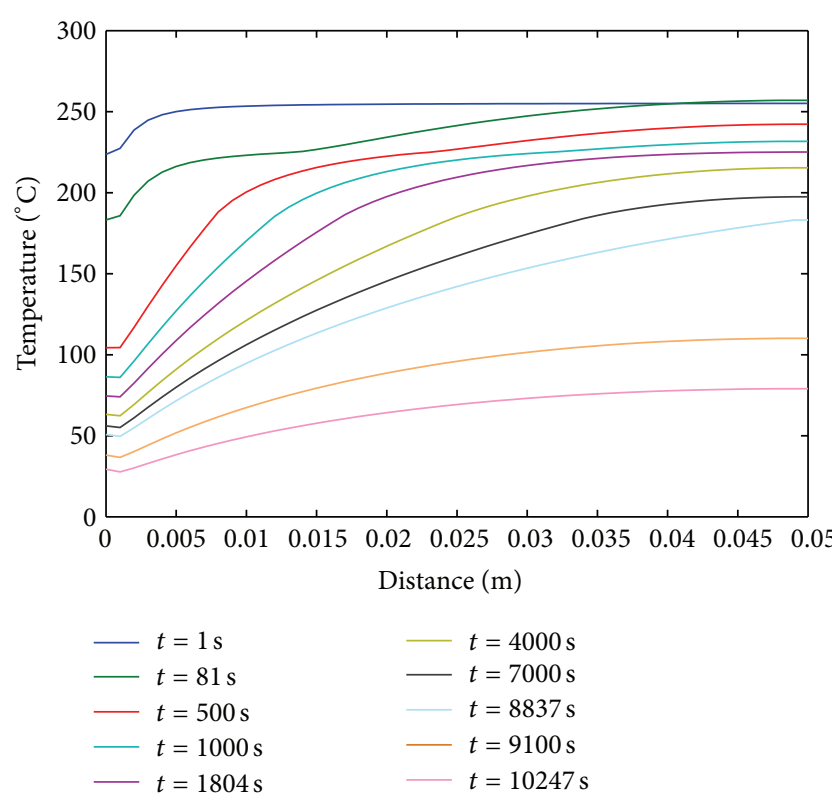

FIGURE 6: Temperature profile in region at different time $t$ for $\alpha=$ 1.6 .

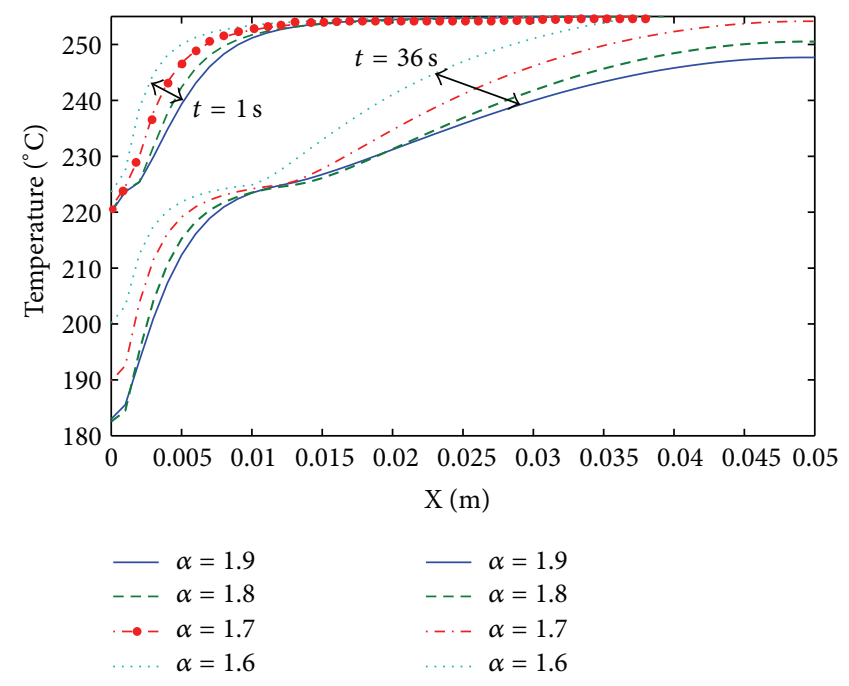

Figure 7: Temperature profile for different $\alpha$ at $t=1$ second and $t=36$ seconds.

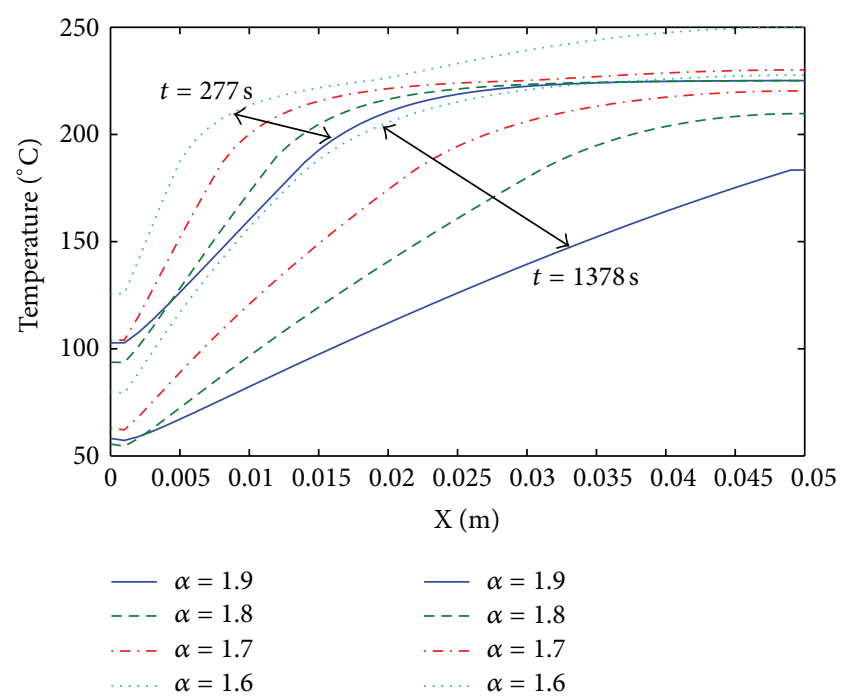

FIGURE 8: Temperature profile for different $\alpha$ at $t=277$ seconds and $t=1378$ seconds.

Figure 5 shows the temperature profile for $\alpha=1.7$ at $t=$ 0.5 seconds, $t=48$ seconds, $t=200$ seconds, $t=537$ seconds, $t=1500$ seconds, $t=2500$ seconds, $t=3805$ seconds, $t=$ 3900 seconds, $t=4000$ seconds, and $t=4739$ seconds. Stage 1 


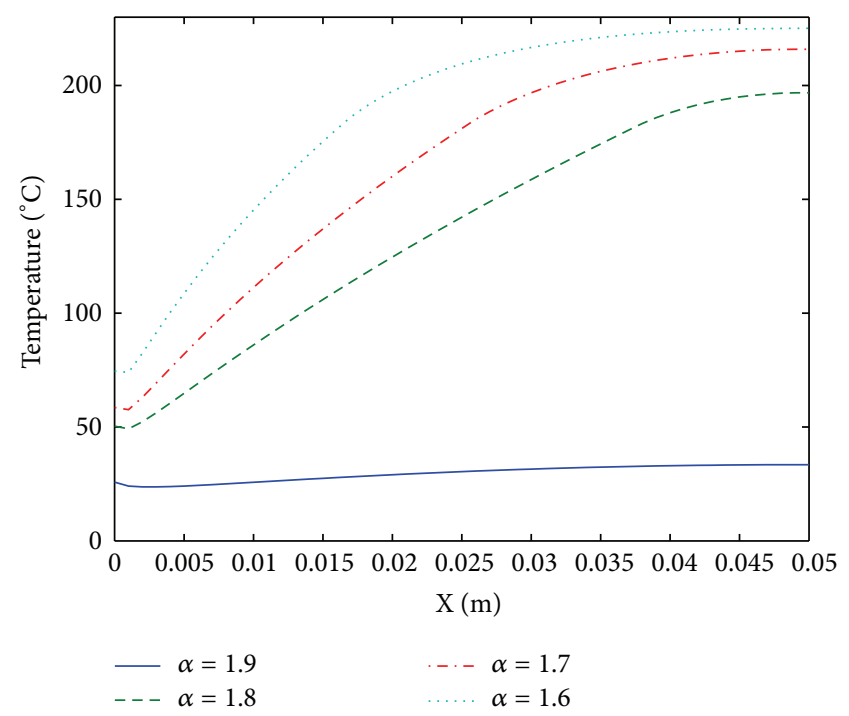

FIgURE 9: Temperature profile for different $\alpha$ at $t=1812$ seconds.

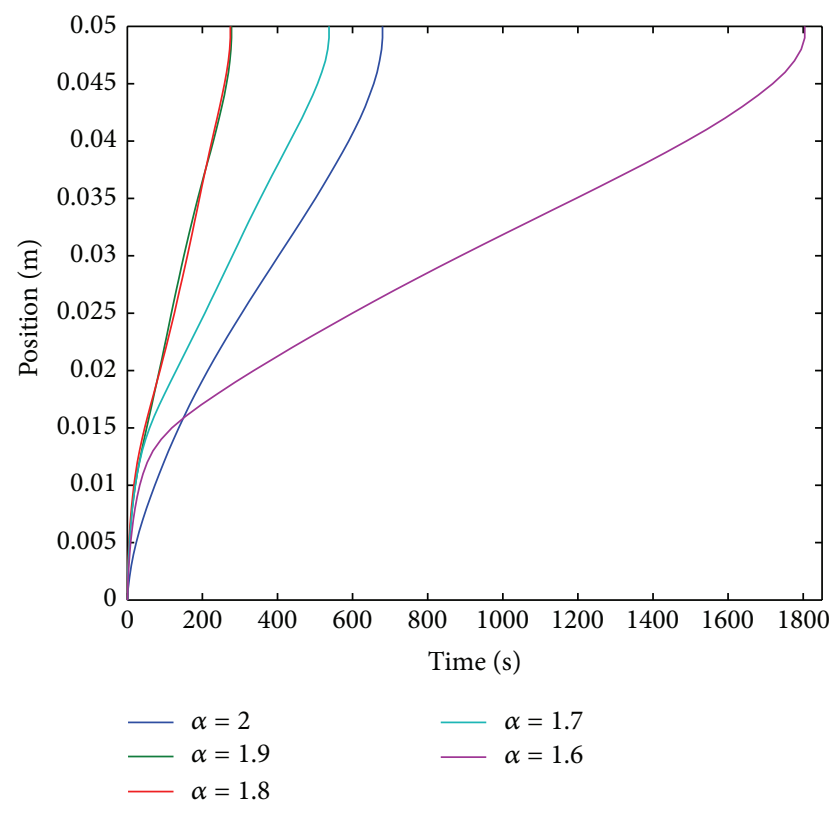

Figure 10: Liquidus interface for different $\alpha$.

ends at $t=0.5$ seconds, when the mushy state starts at $x=0$. At $t=200$ seconds, the system enters into stage 2; that is, the coexistence of mushy and liquid phases. Stage 2 ends at $t=537$ seconds, while stage 3 starts at $t=48$ seconds. Here stage 3 starts before the end of stage 2 . At $t=1500$ seconds, and there exist solid mushy and liquid states. At $t=2500$ seconds, we observe the solid and mushy phases, that is, the system is in stage 3 . Stage 3 ends at $t=3805$ seconds, when the solidification takes place in the entire region. At $t=3900$ seconds and $t=4000$ seconds, the system is in stage 4 ; it ends when it attains its steady state at $t=4739$ seconds.

Figure 6 shows the temperature profile for $\alpha=1.6$ at $t=1$ second, $t=81$ seconds, $t=500$ seconds, $t=1000$

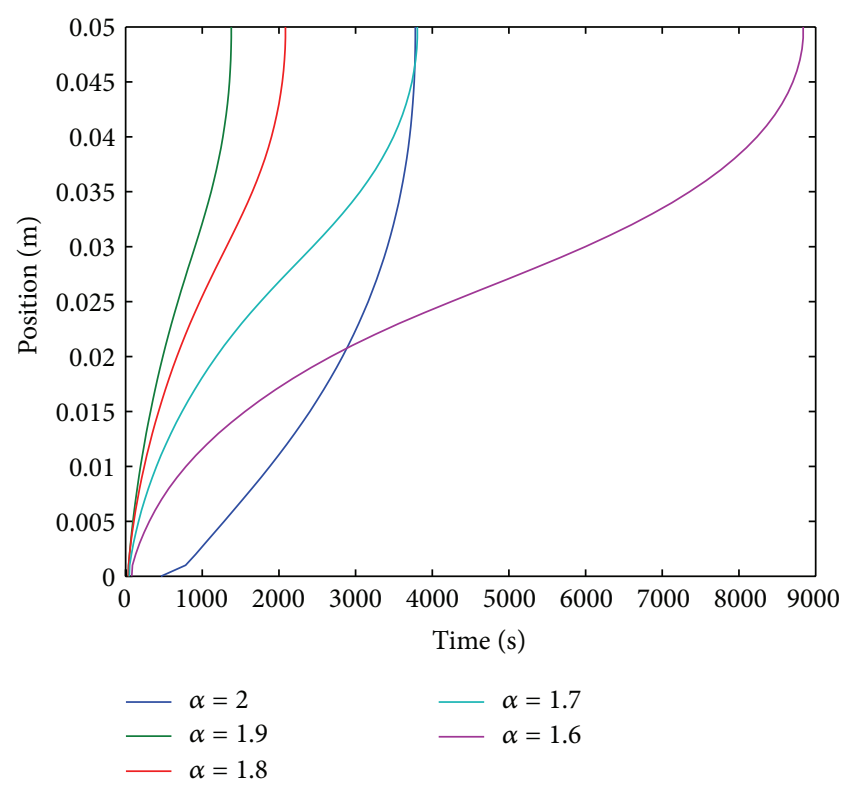

FIGURE 11: Solidus interface for different $\alpha$.

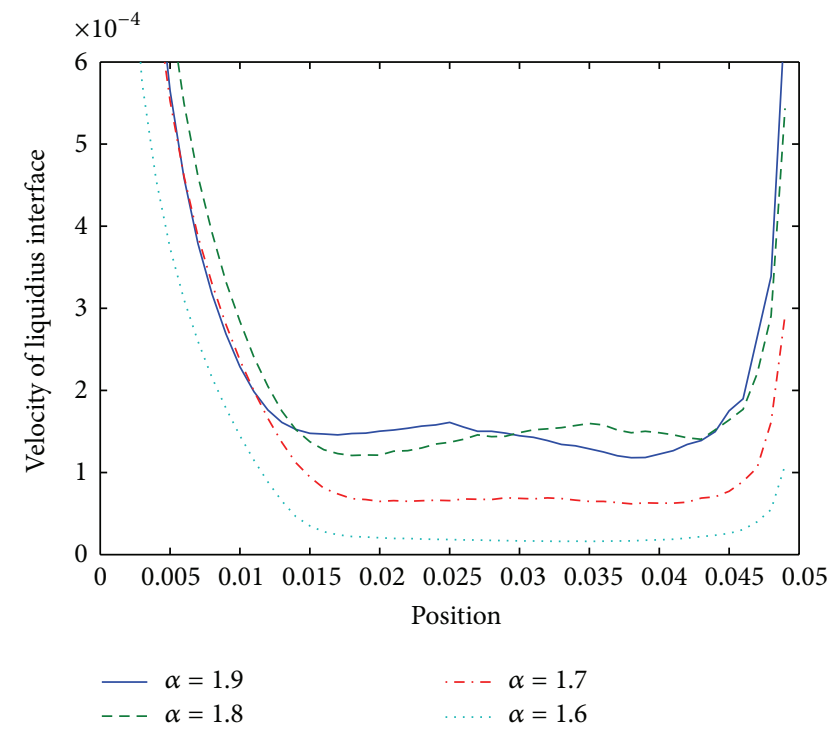

FIGURE 12: Velocity of liquidus interface at interior point for different $\alpha$.

seconds, $t=1804$ seconds, $t=4000$ seconds, $t=7000$ seconds, $t=8837$ seconds, $t=9100$ seconds, and $t=10247$ seconds. Stage 1 ends at $t=1$ second, when the mushy state starts at $x=0$. At $t=500$ seconds, the system is in stage 2 ; that is, the coexistence of mushy and liquid phases. Stage 2 ends at $t=1804$ seconds, and process of stage 3 starts at $t=81$ seconds. Here stage 3 starts before the end of stage 2 . At $t=1000$ seconds, and there exist solid mushy and liquid states. At $t=4000$ seconds and $t=7000$ seconds, we observe the solid and mushy phases; that is, the system is in stage 3 . Stage 3 ends at $t=8837$ seconds, when the solidification takes place in the entire region. At $t=9100$ second the system is in stage 4; it ends when it attains its steady state at $t=10247$ 


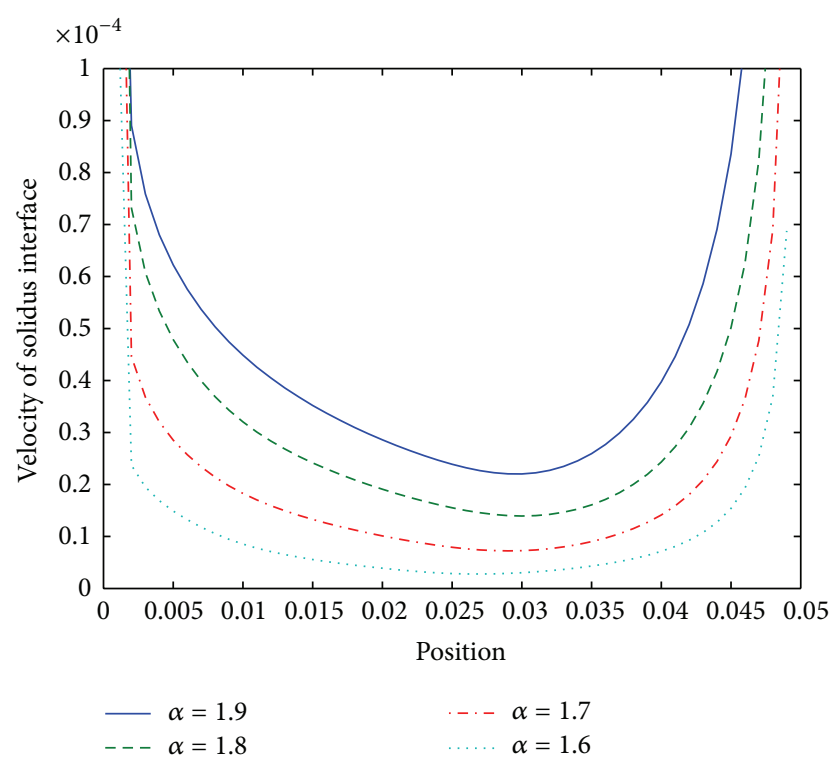

FIGURE 13: Velocity of solidus interface at interior point for different $\alpha$.

seconds. Table 2 represents the comparison of the ends of different stages for different values of $\alpha$.

Figures 7, 8, and 9 exhibit the temperature profile for time $t=1$ second, $t=36$ seconds, $t=177$ seconds, $t=1378$ seconds, and $t=1812$ seconds, respectively. On comparing the temperature for different values of $\alpha$ at fixed time, we observe that temperature is lower in a system for $\alpha=1.9$ as compared to $\alpha=1.8,1.7$, and 1.6.

Figure 10 shows the position of liquidus interface for different values of $\alpha$. We see that interface reaches the end point $x=0.05$ meter for different values of the $\alpha$, and for $\alpha=2,1.9,1.8,1.7$, and 1.6 interface takes 679 seconds, 277 seconds, 274 seconds, 537 seconds, and 1804 seconds, respectively. Figure 11 shows the position of solidus interface for different values of $\alpha$. This takes 3779 seconds, 1378 seconds, 2084 seconds, 3805 seconds, and 8837 seconds, respectively, to reach interface at the end point $x=0.05$ meter for different values of $\alpha$ as $\alpha=2,1.9,1.8,1.7$, and 1.6. It has been observed that interfaces take less time to reach the end point for $\alpha=1.9,1.8$, and 1.7 as compared to $\alpha=2$.

Figures 12 and 13 exhibit the velocity of liquidus and solidus interface, respectively. We observe that the velocity of interfaces increases as $\alpha$ increases.

\section{Conclusion}

In this study, we observe the following.

(i) The system takes less time to attain the steady state for $\alpha=1.9$ and 1.8 as compared to $\alpha=2$; that is, normal diffusion.

(ii) Solidification starts earlier for $\alpha=1.9$ and 1.8 as compared to $\alpha=2$; that is, normal diffusion. (iii) Solidus and liquidus interfaces take less time to reach and the end point for $\alpha=1.9,1.8$, and 1.7 as compared to $\alpha=2$.

(iv) The velocity of the solidus and liquidus is higher at $\alpha=1.9$ as compared to $\alpha=1.8,1.7$, and 1.6.

Looking at the above observations, we can say that the normal diffusion takes more time to attain the steady state position whereas liquidus and solidus interfaces also take more time to reach the end point as compared to fractional diffusion model for $\alpha=1.9,1.8$, and 1.7.

\section{Nomenclature}

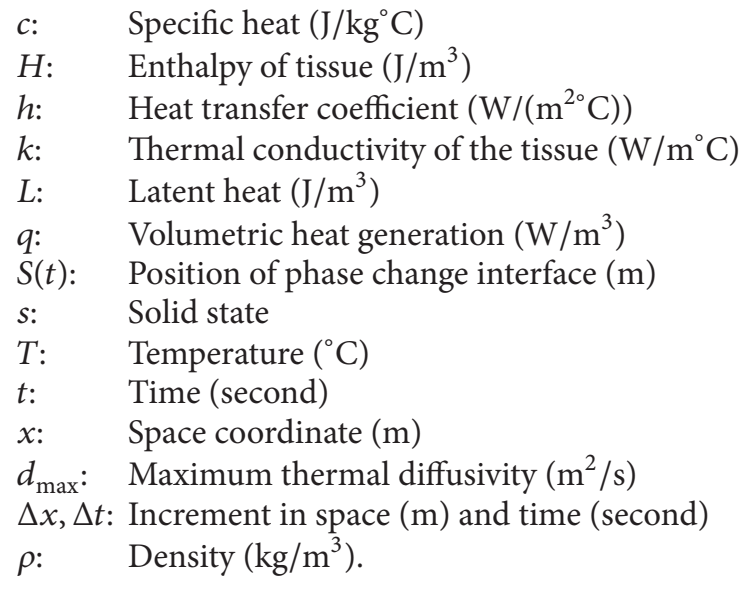

\section{Acknowledgments}

The authors are thankful to the reviewers for their valuable suggestions for improvement of the paper. The first author is thankful to Technical Education Department, Government of Gujarat, India, for providing the opportunity to carry out the research work under quality improvement programme at SVNIT, Surat, India.

\section{References}

[1] J. Stefan, "Ueber die theorie der eisbildung, insbesondere ber die eisbil-dung im polarmeere," Annalen Der Physik Und Chemie, vol. 42, pp. 269-286, 1891.

[2] S. Kumar and V. K. Katiyar, "Transient analysis of alloy freezing in finite media with energy generation and convective cooling," International Journal of Applied Mechanics and Engineering, vol. 15, no. 4, pp. 1155-1168, 2010.

[3] I. Podlubny, Fractional Differential Equations, Academic Press, New York, NY, USA, 1999.

[4] F. Mainardi, "Fractional calculus: some basic problems in continuum and statistical mechanics," in Fractals and Fractional Calculus in Continuum Mechanics, A. Carpinteri and F. Mainardi, Eds., pp. 291-348, Springer, New York, NY, USA, 1997.

[5] M. M. Meerschaert, H. P. Scheffler, and C. Tadjeran, "Finite difference methods for two-dimensional fractional dispersion equation," Journal of Computational Physics, vol. 211, no. 1, pp. 249-261, 2006.

[6] D. A. Murio, "Implicit finite difference approximation for time fractional diffusion equations," Computers and Mathematics with Applications, vol. 56, no. 4, pp. 1138-1145, 2008. 
[7] S. Lijuan, W. Wenqia, and Y. Zhoxima, "Finite difference approximations for the fractional advection-diffusion equation," Physics Letters A, vol. 373, no. 48, pp. 4405-4408, 2009.

[8] M. M. Meerschaert and C. Tadjeran, "Finite difference approximations for fractional advection-dispersion flow equations," Journal of Computational and Applied Mathematics, vol. 172, no. 1, pp. 65-77, 2004.

[9] X. Li, M. Xu, and X. Jiang, "Homotopy perturbation method to time-fractional diffusion equation with a moving boundary condition," Applied Mathematics and Computation, vol. 208, no. 2, pp. 434-439, 2009.

[10] V. R. Voller, "An exact solution of a limit case Stefan problem governed by a fractional diffusion equation," International Journal of Heat and Mass Transfer, vol. 53, no. 23-24, pp. 56225625, 2010.

[11] C. Atkinson, "Moving boundary problems for time fractional and composition dependent diffusion," Fractional Calculus and Applied Analysis, vol. 15, pp. 207-221, 2012.

[12] J. Crank, Free and Moving Boundary Problems, Claredon Press, Oxford, UK, 1984.

[13] H. S. Carslaw and J. C. Jaeger, Conduction of Heat in Solids, Oxford University Press, London, UK, 2nd edition, 1959.

[14] V. Alexiades and A. D. Solomon, Mathematical Modelling of Melting and Freezing Process, Hemisphere, Washington, DC, USA, 1993.

[15] N. Shamsundar and E. M. Sparrow, "Analysis of multidimensional conduction phase change via the enthalpy model," ASME Journal of Heat Transfer, vol. 97, no. 3, pp. 333-340, 1975.

[16] V. R. Voller, "An implicit enthalpy solution for phase change problems: with application to a binary alloy solidification," Applied Mathematical Modelling, vol. 11, no. 2, pp. 110-116, 1987.

[17] C. Bonacina, G. Comini, A. Fasano, and M. Primicerio, "Numerical solution of phase-change problems," International Journal of Heat and Mass Transfer, vol. 16, no. 10, pp. 1825-1832, 1973.

[18] N. E. Hoffmann and J. C. Bischof, "Cryosurgery of normal and tumor tissue in the dorsal skin flap chamber: part I-thermal response," ASME Journal of Biomechanical Engineering, vol. 123, no. 4, pp. 301-309, 2001.

[19] L. M. Jiji and S. Gaye, "Analysis of solidification and melting of PCM with energy generation," Applied Thermal Engineering, vol. 26, no. 5-6, pp. 568-575, 2006.

[20] S. Kumar and V. K. Katiyar, "Numerical study on phase change heat transfer during combined hyperthermia and cryosurgical treatment of lung cancer," International Journal of Applied Mathematics and Mechanics., vol. 3, no. 3, pp. 1-17, 2007.

[21] I. L. Ferreira, J. E. Spinelli, J. C. Pires, and A. Garcia, "The effect of melt temperature profile on the transient metal $/$ mold heat transfer coefficient during solidification," Materials Science and Engineering A, vol. 408, no. 1-2, pp. 317-325, 2005.

[22] V. K. Katiyar and B. Mohanty, "Transient heat transfer analysis for moving boundary transport problems in finite media," International Journal of Heat and Fluid Flow, vol. 10, no. 1, pp. 28-31, 1989. 


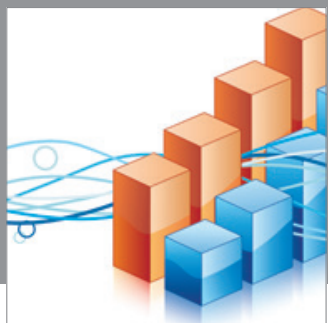

Advances in

Operations Research

mansans

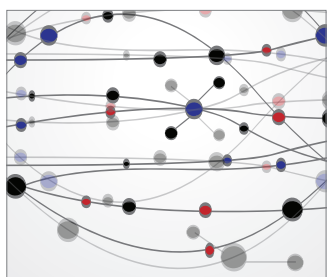

The Scientific World Journal
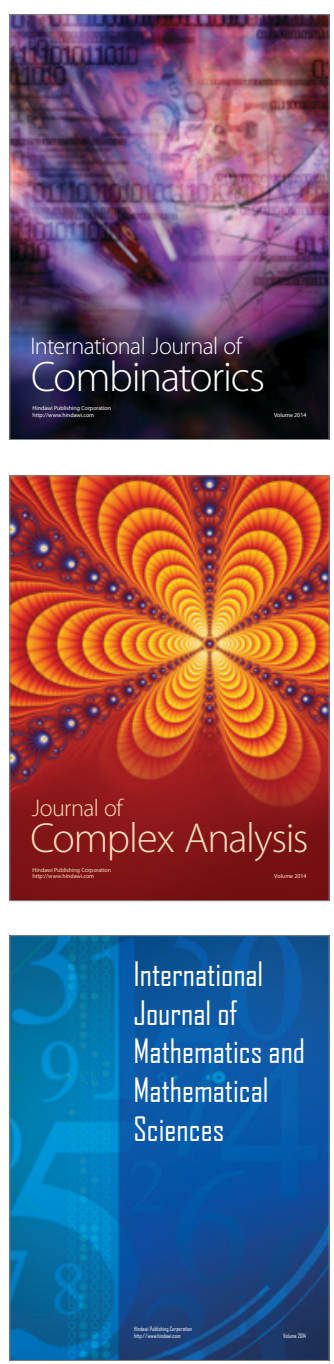
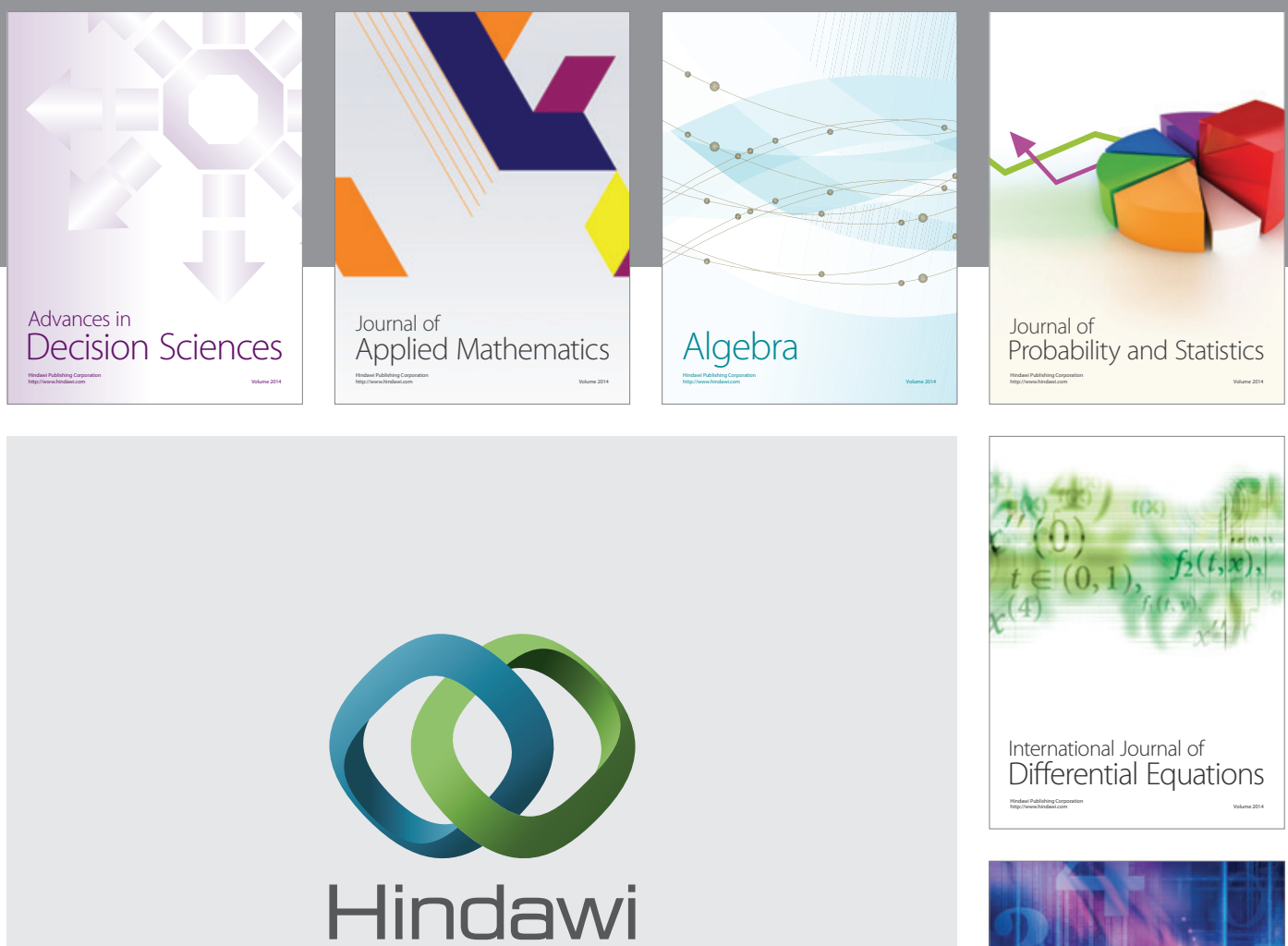

Submit your manuscripts at http://www.hindawi.com
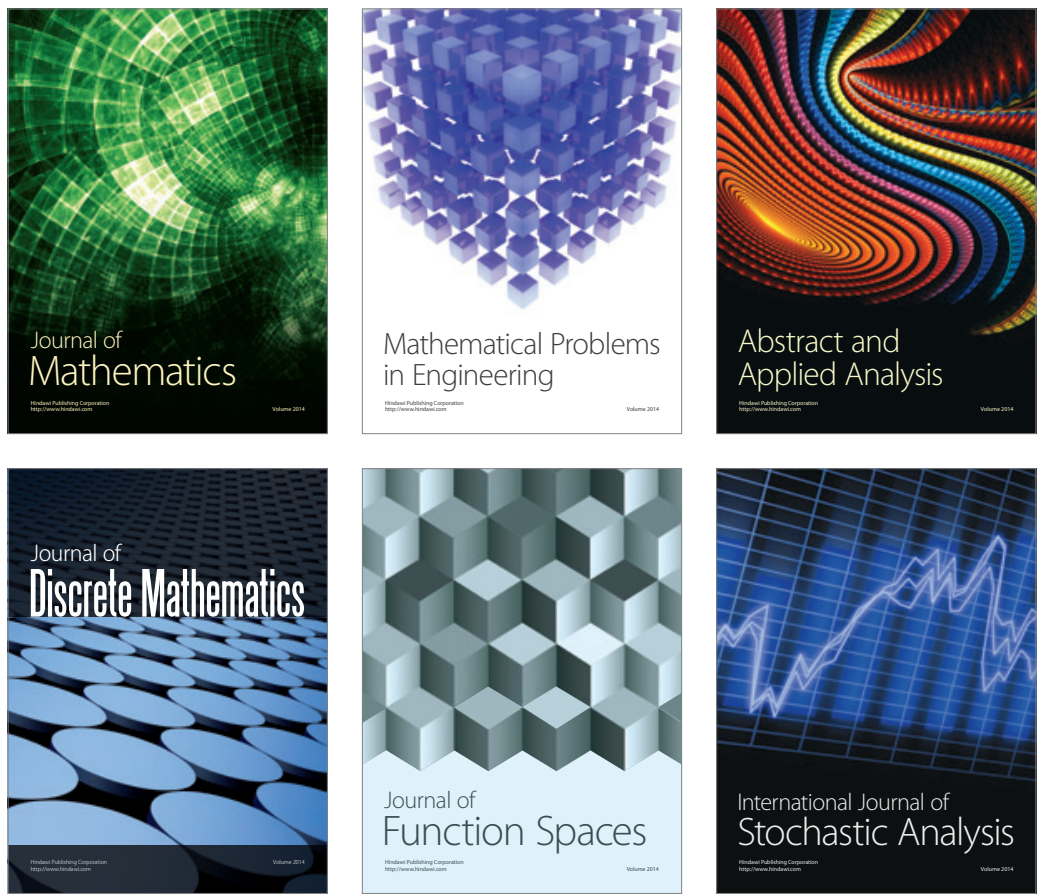

Journal of

Function Spaces

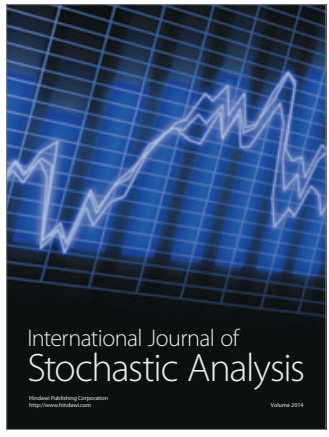

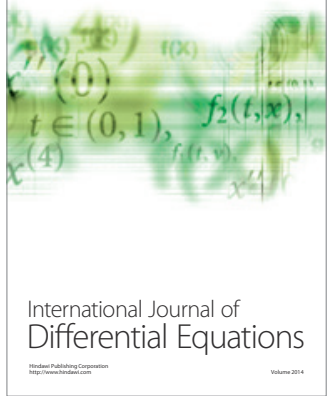
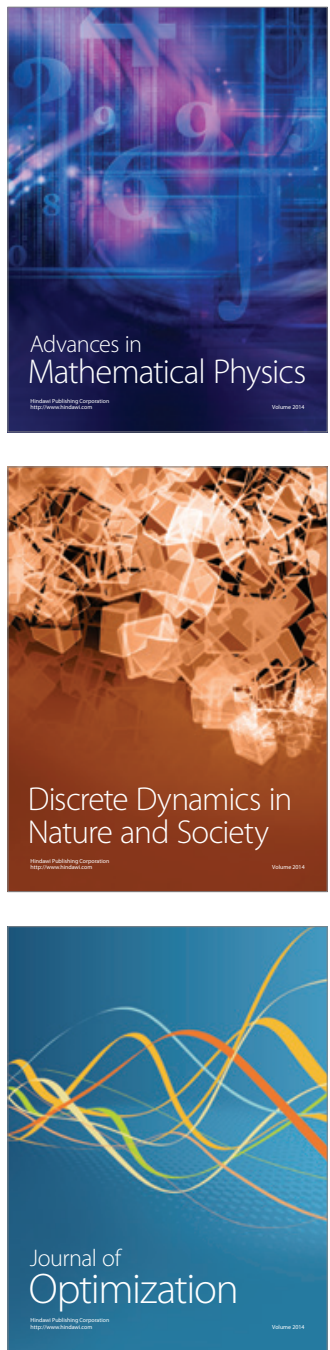